Provided for non-commercial research and education use. Not for reproduction, distribution or commercial use.

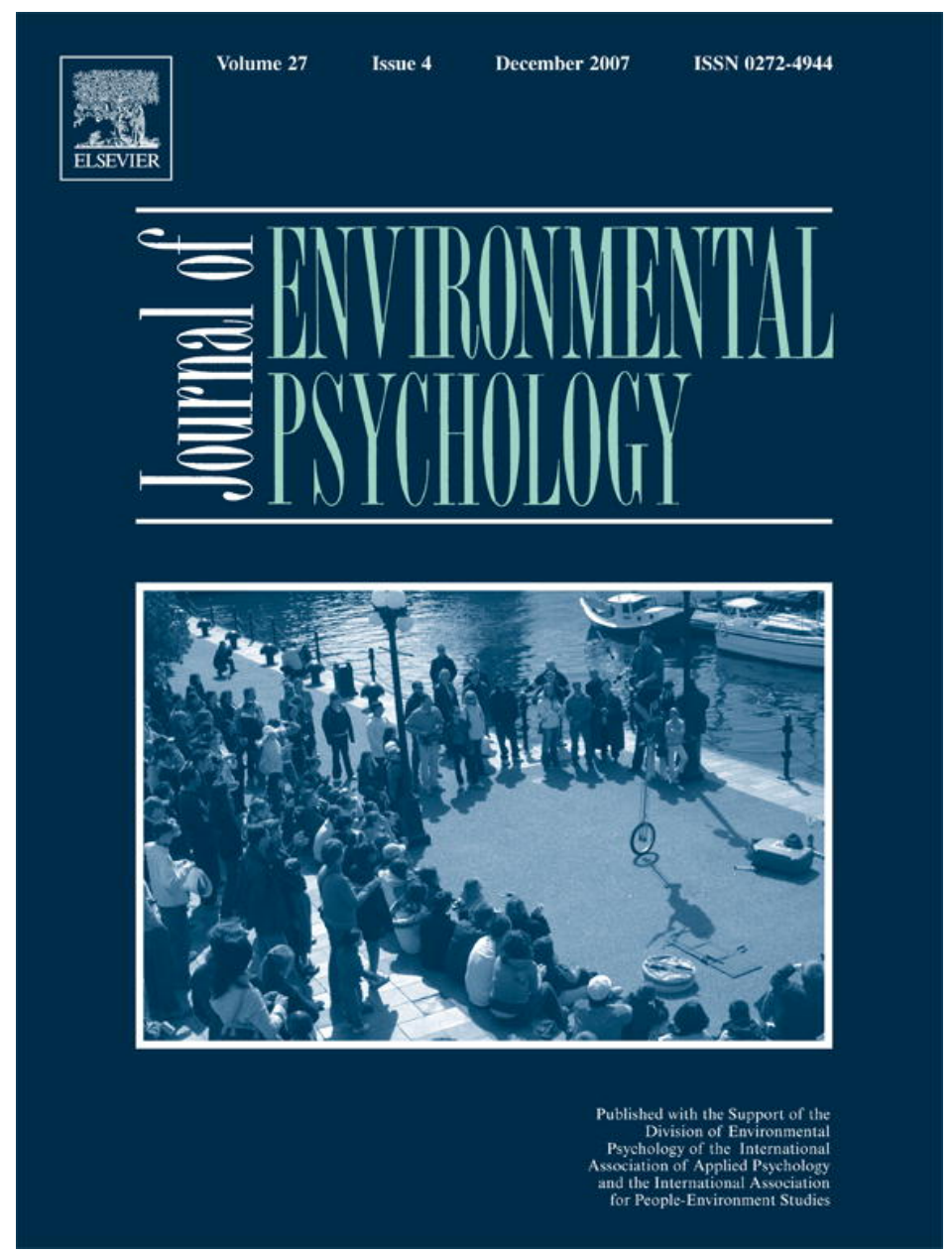

This article was published in an Elsevier journal. The attached copy

is furnished to the author for non-commercial research and education use, including for instruction at the author's institution, sharing with colleagues and providing to institution administration.

Other uses, including reproduction and distribution, or selling or licensing copies, or posting to personal, institutional or third party websites are prohibited.

In most cases authors are permitted to post their version of the article (e.g. in Word or Tex form) to their personal website or institutional repository. Authors requiring further information regarding Elsevier's archiving and manuscript policies are encouraged to visit: 


\title{
Self-construal predicts environmental concern, cooperation, and conservation
}

\author{
Steven Arnocky ${ }^{\mathrm{a}, *}$, Mirella Stroink $^{\mathrm{b}}$, Teresa DeCicco ${ }^{\mathrm{c}}$ \\ ${ }^{a}$ Department of Psychology, Neuroscience, and Behaviour, McMaster University, Canada \\ ${ }^{\mathrm{b}}$ Department of Psychology, Lakehead University, Canada \\ ${ }^{\mathrm{c}}$ Department of Psychology, Trent University, Canada
}

Available online 10 July 2007

\begin{abstract}
The purpose of this study was to determine whether individual differences in self-construal predict differences in environmental concern, resource sharing, and pro-environmental behaviour. University students completed measures of environmental concern, cooperation, and competition in sharing environmental resources, and conservation behaviour. It was found that the independent selfconstrual uniquely predicted egoistic environmental concern and competitiveness in sharing resources. The interdependent self-construal predicted resource cooperation, and the metapersonal self-construal uniquely predicted biospheric environmental concern, ecological cooperation, and self-reports of environmental conservation behaviour. These results suggest that self-construal plays a key role in predicting important environmental thoughts and behaviours. It is proposed that one's environmental concern stems partly from selfconstrual. Quite simply, if one considers the self to include all living things, then one is less likely to harm and more likely to protect the environment.
\end{abstract}

(C) 2007 Elsevier Ltd. All rights reserved.

Keywords: Self-construal; Environment; Cooperation; Environmental behaviour

\section{Introduction}

As the harmful consequences of environmentally destructive human behaviour have become more evident, people worldwide are expressing increased awareness and concern for environmental issues. Although it has been shown that a high percentage of individuals describe environmental problems as being a fundamentally critical social issue (Kempton, Boster, \& Hartley, 1995; Leiserowitz, 2005), many people still view the potential effects of environmental destruction as applying primarily to distant places, individuals, or non-human nature (Leiserowitz, 2005). However, humans also tend to differ greatly in their level of concern for the environment. Some are much more likely to make personal sacrifices to sustain the natural environment than are others. For example, you may view recycling as a necessary procedure, while I may see it as an

\footnotetext{
${ }^{*}$ Corresponding author. Tel.: + 19055259140 ; fax: + 19055296225 .

E-mail address: arnocksa@mcmaster.ca (S. Arnocky).
}

inconvenience or a waste of time. Further, it has been shown that the very reasons underlying our environmental concern differ between individuals. But what are the individual differences affecting environmental concern? Factors such as value basis theory (Schultz \& Zelany, 1999; Stern \& Dietz, 1994), the degree to which people consider themselves part of the environment (implicit connection with nature) (Schultz, 2000; Schultz, Shriver, Tabanico, \& Khazian, 2004), and culture (Milfont, Duckitt, \& Cameron, 2006) have all separately been shown to affect environmental concern and conservation behaviour. Interestingly, these three factors help to determine how human beings construct and perceive their very definition of self, termed self-construal (Markus \& Kitayama, 1991). This study hypothesizes that environmental concern, conservation behaviour, and resource sharing will be predicted by self-construal. Further, we propose that self-construal, which can be conceptualized as consisting of an inclusion of others in self (IOS), cultural influences, and values; will provide a more comprehensive understanding 
of the relationship between the individual and environmental concerns than any of the aforementioned predictors alone.

Indeed, self-construal and concern for the environment share many common features. They both involve a perception held by the individual that is reflected in the priority of their concerns (either for the individual, close others, or all living things). Like environmental concern, perception of the self varies between individuals. The self is created by one's culture, ideals, and practices. It is a dynamic concept that directs an individual's memory, emotional experiences, and perception (Cross \& Madson, 1997). Originally, differences in self-construal have been shown by Markus and Kitayama (1991) on an international level comparing self-construal between North American and Asian cultures. However, individual differences in self-construal also exist within cultures. In examining the hypothesis that self-construal predicts environmental concern, cooperation, and conservation, our study seeks to lend support to the factors identified as influencing environmental concern and also, to offer the perspective that an individual's very definition of selfinfluences their environmental concern, cooperation, and conservation behaviour.

\subsection{Self-construal}

It has been shown that individuals in Eastern cultures such as Asia, construct a definition of the self that is based largely on relationships, and that these individuals place a stronger emphasis on harmony with others than those in Western cultures. Markus and Kitayama (1991) call this the interdependent self-construal. In Western societies, selfconstrual is based on differentiating oneself from others and focussing on the person's unique attributes. This is termed the independent self-construal (Markus \& Kitayama, 1991). Finally, a third model of self-construal has been proposed by DeCicco and Stroink (2007) called the metapersonal self-construal. In this construal, the self is understood to be fundamentally interconnected with all living things; and a person defines his or her self through this unified connection. There is a large amount of research showing the value of self-construal theory for research covering topics such as cognition, emotion, and motivation (Cross \& Madson, 1997). Differences in self-construal are not necessarily culture specific. The independent, interdependent, and meta-personal self-construals each exist in Asian and North American cultures (DeCicco \& Stroink, 2007; Markus \& Oyserman, 1989).

\subsection{Independent self-construal}

Markus and Kitayama (1991) describe the independent self as being comprised of specific morals, traits, abilities, and values that promote self-esteem. Individual behaviour is then a function of these ideological attributes (Markus \& Kitayama, 1991). The thoughts and actions of a person holding an independent self-construal serve to enhance the qualities that make them stand out from others (Markus \& Kitayama, 1991). The independent self-construal is comprised of an emphasis on (a) internal abilities, thoughts, and feelings, (b) being unique and expressing the self, (c) realizing internal attributes and promoting individual goals, and (d) being direct in communication. When thinking about themselves, people with highly developed independent self-construals will refer to their own abilities, attributes, characteristics, or ambitions rather than referring to the thoughts, feelings, or behaviours of others (Singelis, 1994).

\subsection{Interdependent self-construal}

In the interdependent self-construal, one's representations of the self are connected to close others, for example, family relationships. Social contexts and situations must also serve to enhance this view of the self as being generally connected with others (Markus \& Kitayama, 1991). Thus, for a person with an interdependent self-construal, one's self-enhancement is derived from perceptions and emotions that remind them of their connectedness with others. As opposed to the independent self-construal, the interdependent individual has open boundaries between the self and others, and significant relationships with others help to define the self (Cross \& Madson, 1997).

Singelis (1994) states that a person with an interdependent self-construal has a flexible self that emphasizes: (a) external features such as statuses, roles, and relationships, (b) belonging and fitting in with the group, (c) filling one's social role and engaging in appropriate action, and (d) being indirect in communication with others (Singelis, 1994). When individuals with highly developed interdependent self-construals think about themselves or others, there is a sense that the self and others are interconnected (Singelis, 1994).

\subsection{The metapersonal self-construal}

Recently, a third self-construal has been observed, which involves the perception of the self as having a deep interconnection with all forms of life (Stroink \& DeCicco, 2007). This construal differs markedly from the interdependent self, who is only concerned about harmony in relationships held with specific other human beings. Similar to the interdependent and independent self-construals, the metapersonal self-construal is also associated with culture based belief systems such as Buddhism. Indeed, the metapersonal self is very generally associated with the belief systems held by religio-cultural groups like Buddhism and Hinduism (Stroink \& DeCicco, 2007). Stroink and DeCicco (2007) argue that, while these are very different belief systems, they seem to share an understanding of the self that is more accurately described by the metapersonal self-construal than the independent or interdependent self-construals. In these and other 
meditative traditions, the separate self is understood to be an illusion, and the ideal state is believed to be one in which the distinction between subject and object disappears, and all things are experienced as one (Stroink \& DeCicco, 2007).

Since cultural socialization shapes understanding of the self (Triandis, 1989), the degree to which a metapersonal self-construal is held within an individual differs across various cultural groups. As previously mentioned, members of religio-cultural groups such as Buddhism are likely to show the metapersonal self-construal as it is associated with their belief systems. However, the metapersonal selfconstrual is not a function of religious belief in general, as it is dependent on the belief that one is interconnected with all things, which is not a part of all religious belief systems. An individual may also hold the metapersonal selfconstrual without belonging to a religious group (Stroink $\&$ DeCicco, 2007). Stroink and DeCicco (2007) conducted a study in which a sample of Buddhists was compared to a sample of Christians. The Buddhist sample scored significantly higher in metapersonal self-construal than did the Christian sample. This is presumed to be due to the belief in a non-self held by Buddhists.

\subsection{The existence of multiple "selves" within an individual}

People include features of all three aspects of self in their self-systems. The predominant self-construal is determined by the cultural and contextual availability and accessibility of each (Stroink \& DeCicco, 2007). Indeed, if one refers back to the aforementioned study where Stroink and DeCicco (2007) investigated self-construal in Buddhists and Christians, the majority of both samples were Caucasian and resided in Canada, a country that is assumed to be primarily individualistic. The main point of these examples is that within any culture, all three selves can exist within an individual, and situational cues determine the momentary accessibility of each, and their influence on the stream of behaviour. This variability in self-construal within a given culture serves as explanation regarding why individuals will express differences in environmental concern and conservation behaviour within the same population. It also supports the literature showing that although the reasoning behind environmental concern may differ between certain cultures, we do not see any overwhelming differences in pro-environmental behaviour across cultures.

\subsection{Environmental concern}

A three part attitudinal structure for environmental concern has been shown to exist within individuals (Stern \& Dietz, 1994). These environmental concerns are characterised by specific environmental attitudes, which are structured upon a value based theory. Stern and Dietz (1994) proposed that a person's general set of values determine their environmental attitude. Specifically, there are three value bases for environmental attitudes: the individual, all people, and all living things (Schultz \& Zelany, 1999). Any particular person can hold one of three types of environmental concern, very generally defined as (1) egoistic: concern for the self in relation to the environment, (2) altruistic: concern for other people in relation to the environment, or (3) biospheric: concern for the biosphere, respectively. These environmental concerns seem to mirror the primary concerns held by the three types of selfconstrual; either for the self, other people, or all living things.

\subsection{Egoistic environmental concerns}

"Egoistic environmental attitudes are based on beliefs about the effect that environmental destruction may have on the individual. Thus, the environment should be protected because I don't want to breathe polluted air, or I don't want to drink dirty water' (Schultz \& Zelany, 1999, p. 255). It has been found that egoistic environmental concerns are positively correlated with self-enhancement (enhancing one's own personal interest) and negatively correlated with self-transcendence (transcending one's selfish concerns and contributing to the well-being of others) (Schultz \& Zelany, 1999).

\subsection{Altruistic environmental concerns}

Social altruistic concerns are based on goals or benefits to humans. Environmental protection is important to someone who holds this attitude because of the long-term consequences it will have for other people. For example, one who holds this attitude may want to preserve the rainforest because they know that its destruction will bring negative effects to future generations of human beings. Altruistic environmental concerns are similar for those found for biospheric concerns. Altruistic concerns have been found to be negatively correlated with self-enhancement and positively correlated with self-transcendence.

\subsection{Biospheric environmental attitudes}

Biospheric environmental attitudes focus on the inherent value of the environment. Those who hold this attitude believe that human beings should not harm nature because we are a part of nature and all species have a right to exist. Therefore, nature has intrinsic rights broader than the survival or best interest of any one species (Schultz \& Zelany, 1999). Contrary to the egoistic view, biospheric environmental concerns are negatively correlated with selfenhancement and positively correlated with self-transcendence (valuing goals not directly linked to the self) (Schultz, 2001).

\subsection{Culture in determining environmental attitudes}

It has been proposed that culture plays an important role in determining one's environmental attitude. Milfont, 
Duckitt, and Cameron (2006) found that although culture predicts differences in the type of environmental concerns held by Asian versus European descent New Zealanders; biospheric concerns held by individuals of either cultural descent predicted pro-environmental behaviour. This is important to the theoretical framework of the current study because although culture can predict differences in environmental concern, individuals can differ greatly in self-construal within a given culture, which helps to explain why individual differences in environmental concern still exist within a given culture.

\subsection{The relationship between environmental concerns and the self}

Schultz (2001) proposes that objects such as plants, animals and other people, are valued because of the degree to which they are included within a person's cognitive representation of the self (Schultz, 2001). Using the Inclusion of Others in Self (IOS) scale, Schultz (2001) found a positive relationship between interconnectedness of self (the overlapping of self schemas with those of another) and biospheric concerns. Perspective taking and empathetic concerns have also been found to correlate positively with biospheric and altruistic environmental concerns. Additionally, experimentally induced perspective taking has been shown to increase biospheric environmental concerns within individuals (Schultz, 2001).

Schultz (2001) proposed that self-enhancement, which correlated positively with the egoistic concerns, reflects an orientation towards self-benefit. Furthermore, he stated that people who score high on self-enhancement do not define other people or other living things within their perspective of self. In contrast, self-transcendence reflects the degree to which a person includes other humans and other living things within their perception of self (Schultz, 2001), and has been found to be correlated with the metapersonal self-construal (Stroink \& DeCicco, 2007). The finding that altruistic concern did not correlate as strongly with self transcendence as did the biospheric concern, suggests that altruistic concerns reflect an intermediate level of inclusion (Schultz, 2001). This could very well mean only including other close individuals such as the family for the altruistic view, but Schultz (2001) did not test any differences between altruistic and biospheric concerns further.

\subsection{Environmental behaviour}

Environmental concern seems to be a widespread phenomenon. Approximately, $80 \%$ of respondents in one American sample identified themselves as "environmentalists." In addition, people verbally endorsed making lifestyle changes in order to protect the environment, even when the changes require personal cost or inconvenience (Gutfeld, 1991). These commitments by the public towards environmental conservation rarely translate into the actual conservation of resources. In fact, many studies have found low correlations between pro-environmental attitude and conservation behaviour (Thompson \& Barton, 1994).

The weak relationship between environmental concern and actual conservation behaviour may be due to the type of environmental concern held by the sample population. For example, because of the human based values held by those with egoistic or altruistic concerns, they are less likely to act in protection of the environment if other human values such as quality of life interfere. However, those who are biospheric will act to conserve even if it involves discomfort, expense, or inconvenience. The result of this is more overall conservation behaviour by those who care about the environment for its own sake (Thompson \& Barton, 1994). This can have great implications for such things as environmental conservation messages, and education programs. Thompson and Barton (1994) suggest that programs that promote a biospheric view of conservation will result in more pro-environmental behaviours.

\subsection{Cooperation in sharing environmental resources}

Cooperation among human beings in sharing environmental resources is often measured using a social dilemma, such as a commons dilemma - a scenario in which one must compete for or share ecological resources with others (Hardin, 1968). If all participants harvest fewer resources on each turn, the final accumulation of resources for each player is greater than if they were to compete with one another by harvesting the full amount (Hardin, 1968). In these scenarios, individuals can differ greatly on their level of cooperation with others in sharing resources depending on one's predominant self-construal.

In a study by Utz (2004) participants were primed with either the independent or interdependent self-construal. They were then asked to participate in a social dilemma. It was found that participants primed with independence showed lower levels of cooperation than participants primed with interdependence (Utz, 2004).

There is a widely accepted view that cooperation in social dilemmas is based on the person's self interest to do so (e.g., Batson, 1994). It has also been argued that a person's cooperation in a commons dilemma may be explained in part by the decision maker's focus on the consequence of the outcome. If one perceives high criticality (the amount to which they attribute the importance of their decision to the group outcome) and feelings of social responsibility toward the group of participants, the person's contributions will be affected (Cremer \& Dijk, 2002). Therefore, it is likely that the interdependent and metapersonal self-construals would predict social responsibility for others. This leads us to question whether these differences in self-interests and social responsibility between self-construals will be predictive of performance in a commons dilemma. 


\section{Hypotheses}

To summarize, this study contains three distinct hypotheses.

\subsection{Hypothesis one}

Self-construal will predict Environmental concern. Contained within this hypothesis are three specific predictions: The independent self-construal is expected to predict egoistic environmental concerns; the interdependent selfconstrual is expected to predict altruistic environmental concerns; and the metapersonal self-construal will predict the biospheric environmental concerns.

\subsection{Hypothesis two}

It is predicted that the metapersonal self-construal will predict more sharing of the commons and lower depletion of natural resources.

\subsection{Hypothesis three}

The metapersonal self-construal and biospheric concerns will predict self-reported environmental behaviour. Further, we believe that biospheric concerns will mediate the relationship between metapersonal self-construal and pro-environmental behaviour.

\section{Method}

\subsection{Participants}

Participants in the current study were 205 students from Lakehead University in Thunder Bay, Ontario. Of these, 63 were males and 142 were females. The age of the students ranged from 17 to 52, with a mean age of 20 .

\subsection{Materials and procedures}

All questionnaires were provided to each participant at the same time in a questionnaire package. Participants completed an informed consent form, and those participants who were under the age of 18 were also given a parental consent form to be completed by their legal guardian. Participants were given the questionnaire package and allowed to return them anonymously to a box once completed. Upon return of the questionnaire package, participants were given a debriefing regarding the goals of the study. Table 1 provides descriptive statistics for each measure.

Self-construal was measured using a Self-Construal Scale (SCS; Singelis, 1994) that includes 15 items measuring independent self-construal with a .68 alpha, and 15 items measuring the interdependent self-construal with a .71 alpha. These items were intermixed with the 10 items of the metapersonal SCS created by DeCicco and Stroink (2007)
Table 1

Descriptive statistics for all measures

\begin{tabular}{llll}
\hline & $N$ & Mean & Std. deviation \\
\hline Independent SC & 205 & 4.9174 & .571 \\
Interdependent SC & 205 & 4.6816 & .590 \\
Metapersonal SC & 205 & 4.6371 & .751 \\
Egoistic concerns & 201 & 5.4453 & 1.260 \\
Altruistic concerns & 204 & 5.6520 & 1.240 \\
Biospheric concerns & 204 & 6.0167 & .992 \\
Competitive & 205 & 2.4305 & .701 \\
Cooperative & 205 & 3.5951 & .775 \\
Ecological cooperative & 205 & 4.1626 & .623 \\
Environmental behaviour & 205 & 3.3062 & .602 \\
Valid $N$ (listwise) & 201 & & \\
\hline
\end{tabular}

which had an alpha of .77 in the present study. A sample item from this scale is, "I see myself as being extended into everything else." Prior research has shown the metapersonal SCS to have adequate convergent and discriminant validity, and low social desirability bias. There were significant inter-correlations between the self-construal measures. Consistent with Decicco and Stroink (2007), the largest correlation was between the independent and metapersonal self-construals, $r=.572, p=.000$. The theoretically predicted relationships that this scale has shown with values, emotions, attitudes, and cognition, demonstrate that a metapersonal understanding of the selfoperates in a way that is consistent with the self-construal framework, but also meaningfully distinct from the independent and interdependent self-construals (DeCicco \& Stroink, 2007; Stroink \& DeCicco, 2007).

The egoistic, social-altruistic and biospheric environmental concerns were measured using Shultz's (2001) 12 item scale. This scale has been used to lend support to the existence of a three-factor model of environmental concern. Scale scores for each factor were found to have acceptable reliability-egoistic items alpha $=.88$, altruistic alpha $=.81$, and biospheric alpha $=.88$.

Cooperation in a commons dilemma question was measured using a situational questionnaire that was completed individually within the questionnaire package. The purpose of developing and using a questionnaire version of the commons dilemma was to eliminate the need to group people together to run a commons dilemma situation. To the best of our knowledge, this is the first questionnaire form commons dilemma to be developed. The questionnaire format creates a situation to allow for competitiveness, cooperation, and ecological cooperation with hypothetical in-group and out-group members.

\subsection{Commons dilemma scale development}

The commons dilemma questionnaire was developed by creating a hypothetical situation in which participants imagined they were a cattle farmer sharing a common field for grazing, similar to the original commons dilemma. In this situation, they were told that they share the common 
land with two other Canadians and three Americans. Each "farmer" has 10 cattle feeding on the land and it is selfsustaining. The participant then read that they could add 5 more cattle without the others knowing and without depleting the resources too badly, however, if everyone did it, the ecosystem would not renew fast enough. They were then asked to rate 10 questions on a 5-point scale (see Appendix). The questionnaire was created to measure competitiveness (items 1, 2, 4, and 5), cooperation (with other items 3, 6, and 9), and ecological cooperation (cooperation for the sake of the ecosystem-items 7, 8, and 10). Factor analysis using a varimax rotation method supported the three-factor model, accounting for $60.80 \%$ of the variance (see Table 2). Chronbach's alphas were as follows: competitiveness, .68, cooperation, .60, and ecological cooperation, .76.

Lastly, self-reported environmental behaviour was measured with a scale consisting of 15 items pertaining to

Table 2

Varimax rotated component matrix for factor analysis of the self-report common dilemma

\begin{tabular}{rlll}
\hline Item & \multicolumn{3}{l}{ Factor } \\
\cline { 2 - 4 } & (1) Competition & (2) Cooperation & (3) Ecological cooperation \\
\hline 1 & .495 & -.472 & -.368 \\
2 & .482 & -.491 & \\
3 & & .821 & \\
4 & .786 & & -.117 \\
5 & .800 & -.109 & .226 \\
6 & -.206 & .694 & .802 \\
7 & -.195 & .208 & .736 \\
8 & -.175 & .121 & .339 \\
9 & & .557 & .838 \\
10 & & & \\
\hline
\end{tabular}

conservation behaviour, 10 of which were developed by Schultz et al. (2005). This measure has been tested for reliability and validity across many Countries. With the additional 5 items added to the scale, reliability was .81 .

\section{Results}

\subsection{Self-construal and environmental concerns}

Multiple regression procedures were used to examine the relationship between self-construal and environmental concern. To test the first hypothesis that self-construal is related to environmental concern, three multiple regressions were calculated in which the self-construal scores were entered simultaneously to predict each of the environmental concerns. As predicted, when comparing self-construal to the three-part model for environmental concern, it was found that the independent self-construal uniquely predicted egoistic environmental concerns, $\beta=.202, p<.01$. The interdependent self-construal did not predict any of the three environmental concerns, while the metapersonal self-construal uniquely predicted biospheric environmental concerns, $\beta=.214, p<.05$ (Table 3 ).

\subsection{Self-construal and the commons dilemma}

The hypothesis that self-construal is related to cooperation and competitiveness in a commons dilemma was tested using multiple regression. The self-construal scores were entered simultaneously to predict each subscale within the commons dilemma scale (Table 4). The results confirmed the hypotheses in that the independent self-construal predicted competitive attitudes in the commons dilemma, $\beta=.212, p=.012$. In addition, the metapersonal selfconstrual (negatively) predicted competitive attitudes in the

Table 3

Results of three linear regressions showing the relationship between self-construal and egoistic, social altruistic and biospheric environmental concerns

\begin{tabular}{|c|c|c|c|c|c|c|}
\hline \multirow[t]{2}{*}{ Independent variable } & \multicolumn{2}{|c|}{ Regression 1: DV: egoistic } & \multicolumn{2}{|c|}{ Regression 2: DV: altruistic } & \multicolumn{2}{|c|}{ Regression 3: DV: biospheric } \\
\hline & $\beta$ & $p$ & $\beta$ & $p$ & $\beta$ & $p$ \\
\hline Independent self-construal & .202 & $.018 *$ & .078 & .356 & .040 & .634 \\
\hline Interdependent self-construal & -.081 & .288 & -.002 & .979 & -.016 & .834 \\
\hline Metapersonal self-construal & -.035 & .704 & .150 & .101 & .214 & $.019 *$ \\
\hline
\end{tabular}

$* p<.05$.

Table 4

Results of three linear regressions showing the relationship between self-construal and levels of cooperation in a commons dilemma

\begin{tabular}{|c|c|c|c|c|c|c|}
\hline \multirow[t]{2}{*}{ Independent variable } & \multicolumn{2}{|c|}{ Regression 1: DV: competitive } & \multicolumn{2}{|c|}{ Regression 2: DV: cooperative } & \multicolumn{2}{|c|}{ Regression 3: DV: ecological-cooperative } \\
\hline & $\beta$ & $p$ & $\beta$ & $p$ & $\beta$ & $p$ \\
\hline Independent self-construal & .212 & $.012 *$ & -.009 & .911 & -.028 & .721 \\
\hline Interdependent self-construal & .062 & .409 & .199 & $.006^{* *}$ & .017 & .812 \\
\hline Metapersonal self-construal & -.323 & .000 & .231 & $.008 * *$ & .395 & $.000^{* * *}$ \\
\hline
\end{tabular}

${ }^{*} p<.05,{ }^{* *} p<.01,{ }^{* * *} p<.001$. 


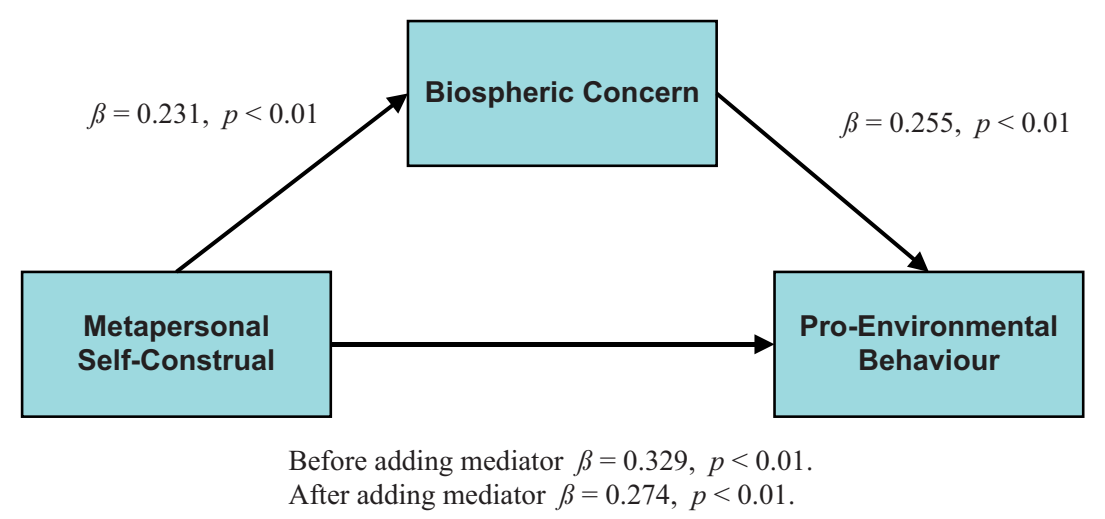

Fig. 1. The relationship between the metapersonal self-construal and environmental behaviour partially mediated by biospheric concern.

commons dilemma, $\beta=-.323, \quad p<.000$. Cooperative attitudes in a commons dilemma were predicted by both the interdependent self-construal, $\beta=.199, p=.006$, and the metapersonal self-construal, $\beta=.231, \quad p=.008$. Finally, the metapersonal self-construal uniquely predicted ecological cooperation in the commons dilemma, $\beta=.395$, $p=.000$.

\subsection{Self-construal, environmental concern, and conservation behaviour}

To test the hypothesis that self-construal would predict ratings of environmental behaviour, a multiple regression was calculated in which the self-construals were entered simultaneously to predict subjective ratings of environmental behaviour. The metapersonal self-construal was the only variable that significantly predicted environmental behaviour ratings, $\beta=.234, p<.01$. It was also found that biospheric environmental concern, when entered into a multiple regression with egoistic and altruistic concern mean scores, was the only environmental concern predictive of self-reported conservation behaviour, $\beta=.306$, $p<.001$.

In determining whether the relationship between the metapersonal self-construal and self-reported conservation behaviour is direct in nature, we examined the potential mediating effect of biospheric environmental concerns on the relationship between metapersonal self-construal and self-reported pro-environmental behaviour. Following the procedure outlined by Baron and Kenny (1986), it was found that each condition for mediation was met. The mediating effect of biospheric concern on the relationship between metapersonal self-construal and pro-environmental behaviour was examined. First, when entered together in predicting environmental behaviour, biospheric concern remained a significant predictor, $\beta=.255, p<.01$, while the metapersonal self-construal score remained significant but was reduced from $\beta=.329, p<.01$, to $\beta=.274, p<.01$. Using the unstandardized regression coefficients, a Sobel test revealed that the relationship between metapersonal self-construal and environmental behaviour was significantly reduced by the inclusion of the biospheric environ- mental concern in the model, $z=2.524, p<.01$. Because biospheric concern acted as a partial mediator, the relationship between the metapersonal self-construal and pro-environmental behaviour is in part accounted for, and thus expressed through biospheric concerns (see Fig. 1).

\section{Discussion}

It was found that, as hypothesized, self-construal was directly related to environmental concern, cooperation, and behaviour. Self-construal predicts a person's reason in caring for the environment. Further, self-construal seems to determine how and why one will behave a certain way in a situation where the individual must share resources, or participate in environmental conservation.

It was also found that the stronger one's independent self-construal, the more egoistic or self-directed their environmental concern. This is opposite to the environmental concern held by those with a metapersonal selfconstrual, who care about the environment for its own sake. This finding contributes to the evidence showing that the metapersonal self-construal is the only definition of self that is concerned with all living things. The latter is suggested as being a more beneficial form of environmental concern as it transcends our concern for the individual or separate self.

Stern and Dietz (1994) proposed that a person's general set of values determine their environmental attitude. These value bases for environmental attitudes are the individual, all people, and all living things. However, culture and the self have also independently been shown to affect environmental concern. The results of this study suggest that because all three of these factors are incorporated in selfconstrual, that self-construal is thereby a more viable determinant of environmental concern than any one factor considered separately. For example, one who is concerned for the environment because they do not want to breathe polluted air, does so not only because they predominantly value the self, but because they actually define the self as being comprised of only the individual. As an opposite example, one who is concerned about deforestation for the sake of the ecosystem and animal life, holds this concern 
not only because they value all living things, but because they define the self as being comprised of all forms of life (and to go against this would in fact be harming the self). From this perspective, environmental concern is heavily related to concern for the self and your reason for concern depends on the contributors to your personal definition of the self.

Self-construal was found to predict ecological cooperation and competition. The finding that the independent self-construal was related to competition when sharing resources confirms that this self-construal is primarily characterized by concern for the individual. The interdependent self-construal and metapersonal self-construal both predicted cooperation with others in sharing resources. However, a further distinction may exist between these two self-construals, in terms of in-group and outgroup ecological cooperation. The current body of research suggests that the interdependent self-construal likely predicts only in-group cooperation and the metapersonal self-construal predicts both in-group and out-group cooperation (Stroink \& DeCicco, 2007). Further, research on social identity processes (specifically, identifying with one's community) has effectively been shown to predict levels of conservation behaviour (Van Vugt, 2001). However, the current version of the questionnaire form commons dilemma was unable to reliably distinguish between in-group and out-group cooperation, and was thus unable to test this hypothesis.

Although both the interdependent and the metapersonal self-construals were significantly related to cooperation, the two self-construals differed in that the metapersonal selfconstrual was related to both cooperation with others as well as ecological cooperation, whereas the interdependent self-construal was related only to cooperation with others. This lends support to our hypothesis that the metapersonal self-construal is concerned for all living things, which includes other individuals in the same way as the interdependent self-construal. The meta-personal self-construal then goes beyond the interdependent self by uniquely predicted ecological cooperation. This involves cooperating with others for the sake of the ecosystem itself, which is characteristic of the self-transcendent values and the nondistinction between the participant and other living things, held by the metapersonal self (Stroink \& DeCicco, 2007). The results clearly show that an individual will compete for resources if they predominantly hold the independent self-construal, and will cooperate with others if they hold either the interdependent self-construal or metapersonal self-construal.

The predicted relationship between the metapersonal self-construal and ratings of environmental conservation behaviour was also found. This relationship is likely due to the metapersonal self-construal incorporating all living things into the definition of the self. Thus, one is likely to care for and actively protect the environment because the entire ecosystem is considered an integral part of the person. Therefore, they are in a way, protecting the self by actively protecting the environment. The non-significant results for the independent and interdependent selfconstruals in predicting pro-environmental behaviour support the idea that we do not see vast differences in pro-environmental behaviour between industrialized independent and collectivist cultures. Similar to Schultz (2001) we suggest that it is not an interconnectedness with other humans, but rather, a consideration of all living things as being a part of the self (an "implicit connection with nature" that is inherent in the metapersonal self-construal) that facilitates a robust expression of pro-environmental behaviour.

Further, the relationship between the metapersonal selfconstrual and pro-environmental behaviour was mediated by biospheric concern. Firstly, this result confirms the conclusion made by Thompson and Barton (1994) that concern for the environment is related to higher levels of conservation behaviour (Thompson \& Barton, 1994). Further, our mediator analyses showed that the relationship between the metapersonal self-construal and proenvironmental behaviour is partially expressed through biospheric environmental concern. Although causal inference cannot be made from this study, the data seem to suggest that the biospheric environmental concern emerges as a function of the metapersonal self, and in turn, predicts pro-environmental behaviour. Environmental conservation programs should broadly promote a metapersonal selfconstrual and more specifically, a biospheric environmental attitude.

\subsection{Directions for future research}

The finding that the interdependent self-construal did not predict any form of environmental concern may have been a Type II error. It is likely that items on the environmental concern scales that measure altruistic concern are simply too specific and do not apply to this particular sample. For example, two of the four items measuring altruistic environmental concern in Schultz's (2001) environmental motives scale ask the participant to rate the importance of both "children" and "my children" in their concern for the environment. It is unlikely; however, that many first year university students have children or are overly concerned with children at this point in their lives. Changing these items to concern for their close friends or family, may have been a more accurate measure of their altruistic environmental concern, and would be more likely to show a significant relationship with the interdependent self-construal.

Future directions for the study of self-construal in relation to environmental cooperation should now focus on whether a primed self-construal can elicit predicted effects in commons dilemma performance. This could have great implications for things such as environmental conservation and awareness campaigns, and may be an effective route to promoting environmental behaviour. For example, experiential education programs, which 
emphasize interconnectedness with the ecosystem may be of value.

The self-report commons dilemma shows potential to be developed into a useful tool for the measurement of ecological cooperation. It is especially useful for situations where it is not feasible to run multiple commons dilemma games. Including items that can distinguish between ingroup and out-group cooperation will be useful in determining differences between the interdependent and metapersonal self-construals. On a more general level, its validity should be assessed by comparing results of the questionnaire to results from a real commons dilemma situation, as there may be subjective differences between what one says they would do and how they actually perform in the situation.

To further explain the relationship between self-construal and environmental behaviours, research should focus on testing whether individuals will actually participate in pro-environmental behaviour as opposed to subjectively rating how often they participate in such behaviours. For example, providing a measure of selfconstrual and then asking participants to sign up for an environmental organization, or to partake in a specific, short environmental activity. This will be crucial to fully establishing the relationship between self-construal and environmental behaviour because it has been shown that even though many people have positive environmental views, these views do not reliably lead to conservation behaviours; and that many studies have found low correlations between environmental attitudes and conservation behaviour (Thompson \& Barton, 1994).

The findings of this study show that self-construal effectively predicts our reasons for environmental concern, as well as how we will cooperate and behave in our use and protection of environmental resources. Further, it is suggested that the reason why we hold a specific concern for the environment and why we behave in certain ways when we interact with the environment, are largely due to concern for the self, and differences depend on what is incorporated in our subjective definition of the self.

\section{Appendix. Self-report commons dilemma}

Imagine that you are a farmer raising cattle. You share grazing land on the Canada-US border with five other people. Specifically, you share the land with two other Canadians and three Americans. You each have 10 cattle feeding off the land and the land renews itself without a problem at these numbers. You have discovered a way to have 5 more cattle feeding off the land without the others knowing, and adding 5 more cattle would not cause too much depletion of the land. Having more cattle on the land will earn you more money, but if everyone were to add 5 more cattle, the land would not renew fast enough, damaging the shared pasture and surrounding ecosystem.
Instructions: Based on the above passage, write the number that best matches your agreement or disagreement, using the 5-point scale below.

\begin{tabular}{lllll}
\hline 1 & 2 & 3 & 4 & 5 \\
\hline $\begin{array}{l}\text { Strongly } \\
\text { disagree }\end{array}$ & Disagree & Neutral & Agree & $\begin{array}{l}\text { Strongly } \\
\text { agree }\end{array}$ \\
\hline
\end{tabular}

In this situation...

1. I would increase my number of cattle on the land without telling the others.

2. I would inform the other two Canadians, but not the Americans.

3. I would inform everyone who I share the common land with.

4. It is important to me that I increase the amount of money I make.

5. It is important to me that I make more money than the other farmers.

6. It is important to me that I cooperate with all of the other farmers.

7. It is important to me that I protect the ecosystem.

8. It is important to me that I practice sustainable farming.

9. I feel responsible for the well-being of all the other farmers.

10. I feel responsible for the well-being of the land and ecosystem.

Competition subscale $=$ items $1,2,4,5$.

Cooperation with others subscale $=$ items $3,6,9$.

Ecological Cooperation subscale $=$ items 7, 8, 10 .

\section{References}

Baron, R., \& Kenny, D. (1986). The moderator-Mediator variable distinction in social psychological research: Conceptual, strategic, and statistical considerations. Journal of Personality and Social Psychology, 51(6), 1173-1182.

Batson, D. (1994). Why act for the public good? Four answers. Personality and Social Psychology Bulletin, 20(5), 603-610.

Cremer, D., \& Dijk, E. (2002). Perceived criticality and contributions in public good dilemmas: A matter of feeling responsible to all? Group Processes \& Intergroup Relations, 5(4), 319-332.

Cross, S. E., \& Madson, L. (1997). Models of the self: Self-construals and gender. Psychological Bulletin, 122(1), 5-37.

Decicco, T., \& Stroink, M. (2007). A third model of self-construal: The metapersonal self. Unpublished manuscript.

Gutfeld, R. (1991). Eight of 10 Americans are environmentalists at least so they say. Wall Street Journal, 218(24), A1-A4.

Hardin, G. (1968). The tragedy of the commons. Science, 162, 1243-1248.

Kempton, W., Boster, J., \& Hartley, J. (1995). Environmental values in American culture. Cambridge, MA: MIT Press.

Leiserowitz, A. (2005). American risk perceptions: Is climate change dangerous? Risk Analysis, 25(6), 1433-1442. 
Markus, H., \& Kitayama, S. (1991). Culture and the self: Implications for cognition, emotion and motivation. Psychological Review, 98, 224-253.

Markus, H., \& Oyserman, D. (1989). Gender and thought: The role of the self concept. In M. Crawford, \& M. Hamilton (Eds.), Gender and thought (pp. 120-127). New York: Springer.

Milfont, T., Duckitt, J., \& Cameron, L. (2006). A cross-cultural study of environmental motive concerns and their implications for proenvironmental behavior. Environment and Behavior, 38(6), 745-767.

Schultz, W. (2001). The structure of environmental concern: For self, other people, and the biosphere. Journal of Environmental Psychology, 21, 327-339.

Schultz, W., Gouveia, V., Cameron, L., Tankha, G., Schmuk, P., \& Franek, M. (2005). Values and their relationship to environmental concern and conservation behavior. Journal of Cross Cultural Psychology, 36.4, 455-475.

Schultz, W., Shriver, C., Tabanico, J., \& Khazian, A. (2004). Implicit connections with nature. Journal of Environmental Psychology, 24, 31-42.
Schultz, W., \& Zelany, L. (1999). Values as predictors of environmental attitudes: Evidence for consistency across 14 countries. Journal of Environmental Psychology, 19, 255-265.

Singelis, T. (1994). The measurement of independent and interdependent self-construals. Personality and Social Psychology Bulletin, 20.5, $580-591$

Stern, P. C., \& Dietz, T. (1994). The value basis of environmental concern. Journal of Social Issues, 50(3), 65-84.

Stroink, M., \& DeCicco, T. (2007). The metapersonal self and cultural belief systems: Implications for cognition. Unpublished manuscript.

Thompson, S. C., \& Barton, M. (1994). Ecocentric and anthropocentric attitudes toward the environment. Journal of Environmental Psychology, 14, 149-157.

Triandis, H. C. (1989). The self and social behavior in differing cultural contexts. Psychological Review, 96, 506-520.

Utz, S. (2004). Self-construal and cooperation: Is the interdependent self more cooperative than the independent self? Self and Identity, 3(3), 177-190.

Van Vugt (2001). Community identification moderating the impact of financial incentives in a natural social dilemma: Water conservation. Personality and Social Psychology Bulletin, 27(11), 1440-1449. 